\title{
A historical review of the concept of labor support in technocratic, humanistic and holistic paradigms of childbirth
}

\author{
Tahereh Fathi Najafi ${ }^{1}$, Robab Latifnejad Roudsari ${ }^{2,3}$, Hossein Ebrahimipour ${ }^{4}$
}

${ }^{1}$ Ph.D. Student of Reproductive Health, Student Research Committee, Department of Midwifery, School of Nursing and Midwifery, Mashhad University of Medical Sciences, Mashhad, Iran

${ }^{2}$ Ph.D. of Reproductive Health, Associate Professor, Evidence-Based Care Research Centre, Mashhad University of Medical Sciences, Mashhad, Iran

${ }^{3} \mathrm{Ph} . \mathrm{D}$. of Reproductive Health, Associate Professor, Department of Midwifery, School of Nursing and Midwifery, Mashhad University of Medical Sciences, Mashhad, Iran

${ }^{4}$ Ph.D. of Health Management, Associate Professor, Social Determinants of Health Research Center, Mashhad University of Medical Sciences, Mashhad, Iran

Type of article: Review

\begin{abstract}
Background: In the past century, maternal support during childbirth has been changed according to the different approaches suggested by various health care paradigms.

Objective: The aim of this review was to argue the maternity supportive care paradigms of the past century and to closely analyze each paradigm.

Methods: This is a historical review, in which published articles were retrieved from databases including Scopus, Science Direct, PubMed and Google Scholar. Sage Journals and Springer's publications were also searched due to the high citation rate of their articles. The keywords entered were "Labor support", "Normal delivery", "Birth attendance", "Supportive approaches", "Health care paradigms", and "Midwifery models of care". They were entered alone or in combinations using "AND". Also, Persian articles were searched in local databases including Irandoc, SID, IranMedex, and Magiran using the above-mentioned keywords in Persian. Sixty articles met inclusion criteria.

Results: The review revealed four main themes including the definitions of continuous labor support, the technocratic paradigm, the humanistic paradigm and the naturalistic paradigm as models of labor support. According to the evidence, labor support has changed from technocratic to humanistic and holistic approaches, which in turn, caused some changes in midwifery models of care used by midwives in the practice.

Conclusion: Labor support based on the holistic approaches and the naturalistic paradigms could bring about remarkable outcomes, the most important being satisfied with the birth experience, increased mother's selfconfidence, enhanced mother's ability in childbirth and better completion of the childbirth process.

Keywords: Supportive Care, Childbirth, Healthcare paradigms
\end{abstract}

\section{Introduction}

From the late $19^{\text {th }}$ century onward, the health system has undergone the most number of transformations (1). The emergence of antibiotics and reduction in the mortality rate led to the Medical Revolution, which shortly after, influenced midwifery care as well, decreasing maternal mortality and morbidity (1). "Medicalization" of pregnancy and childbirth and introduction of interventions into the natural process of childbirth flourished after a few decades; hence, the "Midwifery Renaissance" was born (2). The approach resulting from medicalization was the first approach formed in those years (3). With the emergence of the technocratic paradigm, the bio-medical model of care was developed, which paved the way for transferring childbirth from homes to hospitals, using obstetric tools, taking

\section{Corresponding author:}

Associate Professor, Dr. Robab Latifnejad Roudsari, Mashhad University of Medical Sciences, Mashhad, Iran. Tel: +98.5138597313, Fax: +98.38598016, Email: rlatifnejad@yahoo.com

Received: November 16, 2016, Accepted: June 25, 2017, Published: October 2017

iThenticate screening: June 24, 2017, English editing: August 27, 2017, Quality control: September 16, 2017

This article has been reviewed / commented by three experts

(C) 2017 The Authors. This is an open access article under the terms of the Creative Commons Attribution-NonCommercialNoDerivs License, which permits use and distribution in any medium, provided the original work is properly cited, the use is non-commercial and no modifications or adaptations are made. 
drugs to shorten the childbirth process, reduction of maternal mortality, increase in cesarean sections, and use of medical interventions $(4,5)$. Following the technocratic paradigm, the humanistic and holistic paradigms came into being and the emergence of each of them made drastic changes in maternity supportive care models (6). Recognition of the present paradigms and the resulting healthcare models leads to a thorough assessment of their advantages and disadvantages, which could conduce to their further development (6). Clearly, it triggers detailed and critical analysis of each model which could assist in evaluating the current maternity support models in society. Therefore, by reviewing scientifically sound research studies, we could persuade healthcare policy makers in maintaining or changing the status quo. The authors used a review because it is a specific method to create a comprehensive understanding of a phenomena or a health care problem. The purpose of this historical review is to describe the maternity supportive care paradigms of the past century and closely analyze each of them. By justifying the best paradigm, it aims to persuade health professionals to decide and act accordingly.

\section{Material and Methods}

\subsection{Research design, search strategy, and databases}

The purpose of this historical review is to identify the course of supportive care during labor within different healthcare paradigms over the last century. Databases including Scopus, Science Direct, PubMed and Google Scholar. Sage Journals and Springer's publications were also searched due to the high citation rate of their articles. The keywords entered were the following: "Labor support", "Qualitative study", "Normal delivery", "Birth attendance", "Supportive approaches", "Healthcare paradigms", and "Midwifery models of care". They were entered alone or in combinations using "AND". Also, Persian articles were searched in local databases, e.g. Iran doc, SID, Iran Medex, and Magiran using the above-mentioned keywords in Persian. This process has been shown in the Figure 1.

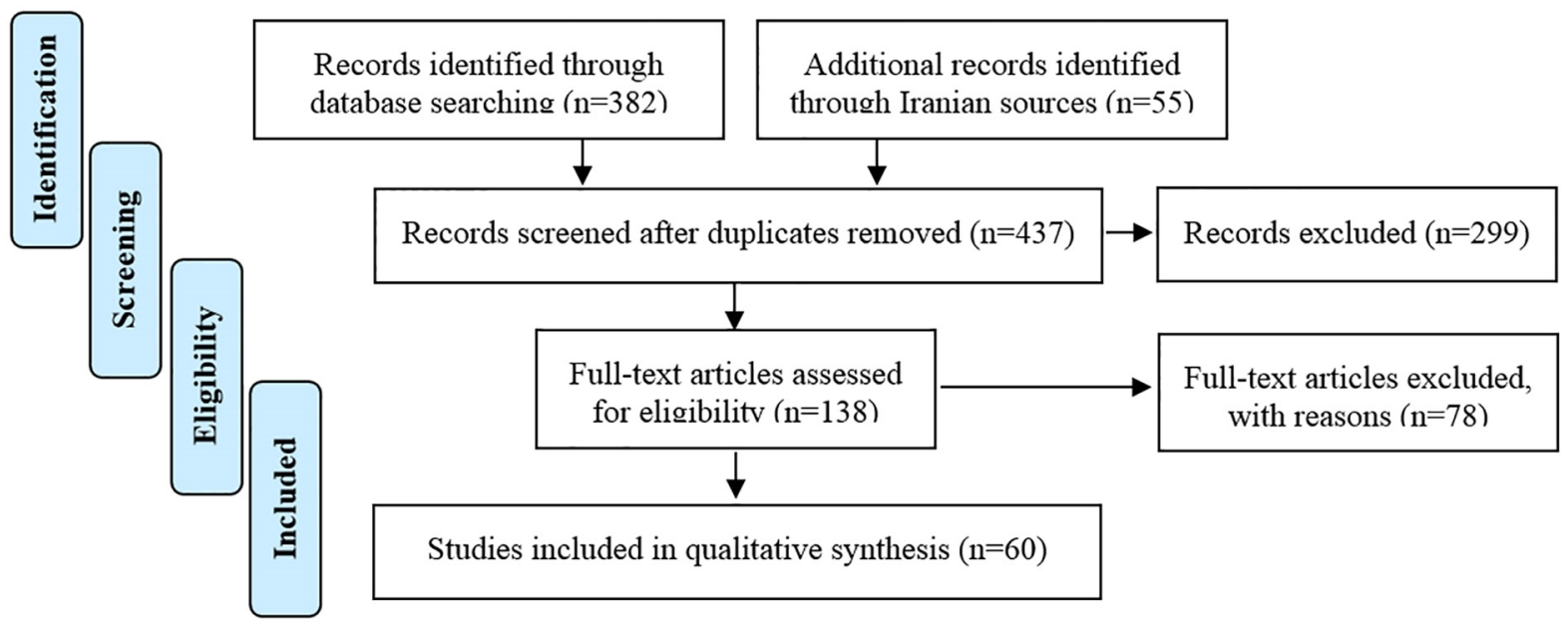

Figure 1. Flow diagram of searching strategy

\subsection{Inclusion and exclusion criteria}

The search was carried out from January through August 2016. The inclusion criteria for selecting articles were the following: considering or discussing maternity support over the last century, being published after 1980 and being written in either English or Persian. The reason for the selection of this particular time period was the identification of changes in healthcare paradigms regarding labor support. In total, 60 articles had the aforementioned criteria. Review of the texts related to support during labor and childbirth revealed that in general, attention to support during labor and childbirth have occurred at two different time points, and changed according to the three healthcare paradigms, which itself, has had a strong impact on supportive care models.

\section{Results}

The review revealed four main themes including definitions of continuous labor support, the technocratic paradigm, the humanistic paradigm and the naturalistic paradigm as models of labor support.

\subsection{Definitions of Continuous Labor Support}

The term "continuous labor support" was first introduced by Bryanton in 1994. At first, Bryanton considered labor support as a type of social support (6). Subsequently, Kenell defined the term as the emotional support provided 
during childbirth (7). Later on, Hodnett (8) presented other definitions of support, specifically, physical, informational, psychological, and emotional. From then on, a supporter was a person who could emotionally empathize with the mother, provide the necessary education to her during labor, support the woman's partner, and offer technical measures to decrease labor pain (8). Overall, the concept of support is derived from Kahn's Theory. Some have divided Kahn's social support and labor-support behaviors into three groups: affect, affirmation and aid. Later, Simkin (1982) defined affect as expressions of admiration, respect and love. Affirmation includes respecting the rights of the mother in her birth plan and supportive behaviors in childbirth and finally, aid is defined as the provision of technical assistance, such as relaxation techniques, breathing and pushing (9-11). Some have also defined support as continuous presence of the attendant beside the mother, encouragement of the mother during childbirth, and provision of necessary instruction. Others have used the term "advocacy" instead of "support". However, Hodnett defined advocacy as the couple's desire to articulate their wishes about the birth plan and to persuade the hospital staff to fulfill those wishes (8). Meanwhile, Simkin stated that labor support consists of various behaviors, i.e. continuous presence, permitting the mother to participate in decision-making, physical examination, instruction, comfort measures, and general care. Labor support has undergone vast changes in different healthcare paradigms (9). Bahri et al. showed a relationship between behavioral and physiological responses due to continuous labor support in Iranian women (12).

\subsection{The Technocratic Paradigm and the Bio-medical Model of Labor Support}

The changes made in the technocratic paradigm are grounded in the western medical system. The main values underlying the technocratic paradigm of medicine are separation of mind and body, mechanization of the human body and inattention to the cultural backgrounds of people (3). The aforementioned issues each have their own outcomes. In this paradigm, the mother's body is like a machine which sometimes becomes defective. Thus, in order to minimize risks, she has to give birth in an equipped factory called the hospital. This line of thought has formed the philosophy of modern midwifery care. The hierarchical system in this paradigm sees the doctor as the authority and cues, such as white coats and technical jargon, add to this status. Therefore, the patient lacks responsibility and the practitioner, as the authority, is the only person responsible. The technical paradigm and bio-medical model believe in the separation of the human body from the human mind and consider childbirth as a separate experience from the flow of life. This approach led to advances in the physiopathological diagnosis of disorders during pregnancy. However, due to its mechanical view of the body, it perceived the process of childbirth as mechanical, and drew all the attention of the practitioner to the physical changes of the mother during pregnancy and labor $(1,3$, 4). The technical paradigm and its resulting medical model, led to gradual removal of humanistic values, decrease in women's ability to bear and give birth to a child, increase in the rate of cesarean sections, and women's blind acceptance of the doctor's decision; and therefore, are currently not followed in many developed countries $(1,13$, 14). In this approach, along with technological advances in healthcare, the opportunity was lost for the midwife to touch the childbearing woman. The close distance of the midwife with the mother and the skin contact between them gave their place to electronic monitoring devices $(15,16)$. Gradually, with the emergence of diagnostic tools such as ultrasonography, using them became common (16). The concept of presence beside the mother for better care provision during childbirth, was slowly eliminated (16). The contribution of this approach was a decrease in maternal and neonatal mortality and morbidity (4). According to Kirkham and Stapleton, the results of the mentioned developments in the bio-medical model reinforce the values of obstetrics, not those of midwifery (17). The results of the first meta-analysis on continuous labor support revealed that continuous labor support by a labor attendant shortens the duration of labor by 2.8 hours and significantly decreases the use of obstetric equipment (18). However, Hodnett mentions the impracticality of support by relatives in the labor unit and the delivery room, and in a systematic review study, points out that the presence of a doula and an attendant is possible in high-income countries with high social capital; however, it is not practically possible in low-income countries. On the other hand, Hodnett states that the woman's partner and family members are not able to fulfill a good supporting role because firstly, they do not have the required training and secondly, these individuals themselves need support during that time. Hodnett's study reveals that the best supporter in this critical period is a qualified professional, namely a midwife, nurse or healthcare provider $(5,19)$.

\subsection{The Humanistic Paradigm and the Bio-Psycho-Social Model of Labor Support}

The formation of this approach and attention to labor support with a humanistic view towards childbirth, began in the 1970s. The humanistic paradigm entered midwifery care with the purpose of connecting the mind and the body. This approach perceives the treatment of physical illnesses without consideration of psychological factors as practically impossible. It believes in the aggregation of mind and body, and replaces the mechanical view towards childbirth with an organismal one. The greatest achievements of this ideology include changes in the paternalistic 
outlook, in the one-way treatment and decision-making of doctors and health care providers, and finally the formation of shared responsibility between patient and practitioner (20). Technology with humanism, focus on prevention, accepting death as an outcome, compassion-driven care, and open-mindedness towards other modalities. The outcomes of this paradigm led to the formation of the bio-psycho-social model, which emphasizes on the patient-healthcare practitioner relationship, continuous presence of the practitioner during childbirth, careful attention to the patient's words, learning how to communicate with the patient in order to make better diagnosis, allowing patients to express their feelings and concerns, obtaining informed consent and respecting the patient's rights, and permitting the mother to choose her position for child delivery. The central core of studies done in this paradigm was treatment-based, gradually drifting support from some physical actions to emotional and psychological support and their effects on childbirth results. Arulkumaran (21) claimed that emotional labor support not only leads to positive physical outcomes in childbirth, but also provides a pleasant experience of childbirth and minimizes the psychological side-effects in the postpartum period. Regarding those who support the laboring woman, Arulkumaran stated that little benefit could be seen with support from the woman's partner or family members. Thoits reviewed empirical work on the buffering hypothesis, and indicated that in order to create a positive outcome out of life events, including childbirth, social support has a much greater effect than reduction of stress-causing factors (22). Studies within this approach have considered many aspects to the concept of support, and redefined different types of support during labor and delivery (23). Adam's and Binachi (2008) grouped support into four categories: physical support, emotional support, instructional/informational support and advocacy. They also subdivided each of the groups within certain frameworks. For example, physical support does not only mean the presence of the midwife beside the mother, but also includes environmental control, creating a comforting atmosphere, hydrotherapy, nourishment, touch and massage, attention to the mother's hygiene, and partner care (24). Despite the positive results of labor support in the bio-psycho-social model, some studies find the real support provided by midwives during labor and delivery unsatisfactory, which they attribute to the obligation of filling out medical record sheets. Basically, they believe that midwives do not provide the required support to mothers. Although support by the midwife leaves the highest effect on creating positive results during and after childbirth, several studies, including Miltner's study, indicate that midwives do not spend enough time for supporting the laboring woman (23).

\subsection{The Naturalistic Paradigm and the Holistic Approach of Labor Support}

The Naturalistic Paradigm, which is a combination of the above-mentioned paradigm, takes into account the mind, body, environment and society all at once. This holistic view of the human being was developed in the recent years. This approach sees health and healing dependent on all these factors, and puts more significance on the mind and metaphysics. Moreover, the body is viewed as an energy field that could connect with other fields of energy (25, 26). The outcome of this approach is that the midwife and the supportive care she provides, could create a vast energy field. Furthermore, the general healing of the patient depends on the healing of all factors influencing her life. Accordingly, illnesses are created by the imbalance of the mind and the body, and in order to heal illnesses, all contextual factors, even the climate of place of residence, are essential (26). The next tenet of this approach is the unity of practitioner and client. Healing starts from the inside-out, and outside factors are brought to attention later (4). Care is individualized according to the patient's condition, and practitioners respond to the unique needs of each patient. For example, the midwife recommends a different method of pain management, consistent with the birthing woman's labor conditions (4). Finally, this paradigm views death as a step in the process of living rather than the final stage. Seeing the body as an energy field, which believes that energy never dies, but enters another process (4).

\section{Discussion}

With changes in healthcare paradigms in all areas including childbirth, the social and cultural setting of women gradually came into consideration in childbirth. Historically and cross culturally, childbirth has been associated with the constant presence of a caregiver. However, since the concept of midwifery has originated from and advanced in European countries, especially England, the supportive care given by the midwife has also undergone many changes in the course of time. The beginning of these changes took place according to the supportive care models; however, from a long time ago the midwife had been offering supportive care to the mother giving birth. By establishing a close rapport with the woman in labor, the midwife provides complete support to the mother. This concept has not been changed in the course of time or with alterations of supportive care models (27). From an earlier period and in all cultures, the presence of an attendant, aside from the midwife, who could be a family member or the woman's partner, was customary (27). It could be claimed that the main ideology behind midwifery in all cultures was the constant support of the mother during the labor and delivery and the word "midwife" has been defined based on this ideology. A midwife who is detached from this ideology experiences psychological tensions, fatigue, depression, job 
dissatisfaction, and burnout. Return to the original identity of this profession in some countries has been made possible by changes in supportive care models (28-30). In recent years, due to changes in caring and healing approaches, family structure, and decrease in the duration of hospitalization, a new wave of supportive care has been formed for pregnancy, labor, childbirth, and breastfeeding (29). Tilingen investigated the essential concepts of childbirth in both the obstetric-model and the midwifery-model, and provided a critical analysis of them (28). Tilingen indicated that in the obstetric-model, the medical approach is more highlighted. According to this model, pregnancy and childbirth are potentially pathological; hence mothers are labeled "high risk" or "low risk". In the midwifery model, on the other hand, childbirth is seen as a natural process, in which individual and social factors play a great role (28). Aside from the two mentioned paradigms, Karlsdottir et al. recognize the "childbearing woman's paradigm" as influential in forming the holistic look at childbirth (29). Karlsdottir et al. claim that the childbearing woman's paradigm mostly focuses on issues such as "who will do the delivery?" and "how will pain be controlled?" Moreover, the supportive care provided by the caregiver during labor could affect the childbearing woman's paradigm (29). The naturalistic studies carried out in Iran also indicate that continuous support during labor and childbirth does not occur due to reasons such as the dominant cultural setting in hospitals, and shortage of personnel $(30,31)$. Taghizadeh et al. attempted to reveal the hidden side of the psychological disorders caused by the childbirth experience in Iranian mothers in a qualitative study (31). The study discusses how lack of continuous support throughout the childbirth process in Iranian hospitals leads to greater fear and anxiety in mothers, and paves the way for psychological birth trauma. Although Iranian women's attitude towards childbearing is not negative, the persistence of helplessness and anxiety during delivery and insufficiency of proper support could bring negative attitudes to them. In view of the remarkable success of Iran in achieving the two health indicators of the millennium development goals, i.e. reducing maternal morbidity and mortality and increasing the number of births supervised by skilled and educated midwives, more attention is required to support Iranian women throughout the childbirth process, and protect them against psychological birth trauma (32-35).

\section{Conclusions}

It is therefore concluded that labor support based on the holistic approach and the naturalistic paradigm could bring about remarkable results, the most important being satisfied with the birth experience, increased mother's selfconfidence, enhanced mother's ability in child delivery and better completion of the childbirth process.

\section{Acknowledgments:}

This study is a part of a doctoral thesis which has been conducted using grounded theory approach. The project has been approved in Mashhad University of Medical Sciences. The funding support of this work was given by the Vice Chancellor for Research, Mashhad University of Medical Sciences (MUMS), Mashhad, Iran (Grant number: 930253). The authors express their gratitude to Mashhad Medical Science University.

\section{Conflict of Interest:}

There is no conflict of interest to be declared.

\section{Authors' contributions:}

All authors contributed to this project and article equally. All authors read and approved the final manuscript.

\section{References:}

1) Shaw JC. The medicalization of birth and midwifery as resistance. Health Care Women Int. 2013; 34(6): 522-36. doi: 10.1080/07399332.2012.736569. PMID: 23514572.

2) Christiaens W, Nieuwenhuijze MJ, de Vries R. Trends in the medicalisation of childbirth in Flanders and the Netherlands. Midwifery. 2013; 29(1): e1-8. doi: 10.1016/j.midw.2012.08.010. PMID: 23266221.

3) Davis-Floyd R. The Technocratic, Humanistic, and Holistic Paradigms of Childbirth. Int J Gynaecol Obstet. 2001: 75(1): s5-s23. doi: 10.1016/S0020-7292(01)00510-0. PMID: 11742639.

4) Arab M, Noghabaei G. Importance of holistic view to medical profession. J Med Educ Develop. 2014; 8(4): 75-8.

5) Hodnett ED, Gates S, Hofmeyr GJ, Sakala C. Continuous Support for Women During Childbirth. BIRTH. 2005; 32: 72-9.

6) Bryanton J, Fraser-Davey H, Sullivan P. Women's perceptions of nursing support during labor. J Obstet Gynecol Neonatal Nurs. 1994; 23(8): 638-44. doi: 10.1111/j.1552-6909.1994.tb01933.x. PMID: 7836988.

7) Kennell J, Klaus M, McGrath S, Robertson S, Hinkley C. Continuous emotional support during labor in a US hospital: a randomized controlled trial. JAMA. 1991; 265(17): 2197-201. doi: 10.1001/jama.1991.03460170051032. PMID: 2013951. 
8) Hodnett ED, Lowe NK, Hannah ME. Continuous labour support by a nurse did not reduce the rate of cesarean delivery. Evidence-based Obstetrics and Gynecology. 2003; 5: 8-9. doi: 10.1016/S1361259X(03)00038-2.

9) Simkin P. Supportive Care During Labor: A Guide for Busy Nurses. JONNG. 2002: 31(6); 721-32.

10) Lazarus S. Thoughts on the relations between emotion and cognition. American Psychologist. 1982; 37(9): 1019-24. doi: 10.1037/0003-066X.37.9.1019.

11) Tumblin A, Simkin P. Pregnant Women's Perceptions of Their Nurse's Role During Labor and Delivery. BIRTH. 2001: 28: 52-6.

12) Bahri Binabaj N, Latif Nejad R, Esmaili H. Study of the effect of continuous maternal support on behavioral and physiologic responses to labor pain. J Sabzevar Univ Med. 2005; 1(11): 24-33.

13) Rosen P. Supporting Women in Labor: Analysis of Different Types of Caregivers. J Midwifery Women's Health. 2004; 49(1): 24-31. doi: 10.1016/j.jmwh.2003.10.013. PMID: 14710137.

14) Compton JD. Women's experiences and expectations of the physician-patient relationship. A Dissertation Presented for the Doctor of Philosophy Degree.The University of Tennessee, Knoxville. 2005.

15) Moscrop A. The Captured Womb: A History of the Medical Care of Pregnant Women. BMJ. 2011; 342: d2531. doi: 10.1136/bmj.d2531.

16) Adams E, Bianchi A. A Practical Approach to Labor Support. J Obstet Gynecol Neonatal Nurs. 2008; 37 : 106-15. doi: 10.1111/j.1552-6909.2007.00213.x. PMID: 18226164.

17) Kirkham M, Stapleton H. Midwives' support needs as childbirth changes. J Adv Nurs. 2000; 32(2): $465-72$.

18) Zhang J, Bernasko J, Fahs M. Countanious Labour Support from labour attendant for primeparous women: A Meta analysis. Obst Gyn. 1996; 88(4): 739-44.

19) Hodnett ED, Gates S, Hofmeyr GJ, Sakala C. Continuous support for women during childbirth. The Cochrane Library. 2013. doi: 10.1002/14651858.CD003766.pub5.

20) Raphael-Leff J. Psychological Processes of Childbearing. Forth Edition. Chapman and Hall; 1991.

21) Arulkumaran S. Psychosocial support or active management of labour or both to improve the outcome of labour. BJOG.1999; 106: 617-9. doi: 10.1111/j.1471-0528.1999.tb08356.x.

22) Thoits PA. Conceptual, Methodological, and Theoretical Problems in Studying Social Support as a Buffer Against Life Stress. J Health Soc Behav. 1982; 23: 145-59. doi: 10.2307/2136511. PMID: 7108180.

23) Miltner R. More Than Support: Nursing Interventions Provided to Women in Labor. J Obstet Gynecol Neonatal Nurs. 2002; 31(6): 753-61. doi: 10.1177/0884217502239214. PMID: 12465872.

24) Adams ED, Bianchi AL. A practical approach to labor support. J Obstet Gynecol Neonatal Nurs. 2008; 37(1): 106-15. doi: 10.1111/j.1552-6909.2007.00213.x. PMID: 18226164.

25) Berg GV, Sarvimäki A. A holistic-existential approach to health promotion. Scand J Caring Sci; 2003; 17; 384-39. doi: 10.1046/j.0283-9318.2003.00240.x. PMID: 14629641.

26) McEvoy L, Duffy A. Holistic practice--a concept analysis. Nurse Educ Pract. 2008; 8. doi: 10.1016/j.nepr.2008.02.002. PMID: 18362085.

27) Marland H. The Art of Midwifery. The third Edition. Taylor \& Francis e-Library; 2005.

28) Teijlingen E. A Critical Analysis of the Medical Model as used in the Study of Pregnancy and Childbirth. Socio Res Online. 2005; 10(2).

29) Karlsdottir S, Halldorsdottir S, Lundgren I. The third paradigm in labour pain preparation and management: the childbearing woman's paradigm. Scand J Caring Sci. 2013; 1-13.

30) Fathi Najafi T, Latifnejad Roudsari R, Ebrahimipour H. The best encouraging persons in labor: A content analysis of Iranian mothers' experiences of labor support. Plos one. 2017; 12(7): e0179702. doi: 10.1371/journal.pone.0179702. PMID: 28683112, PMCID: PMC5499987.

31) Taghizadeh Z, Irajpour A, Nedjat S, Arbabi M, Lopez V. Iranian mothers' perception of the psychological birth trauma: A qualitative study. Iran J Psychiatry 2014; 9(1): 31-6. PMID: 25561946, PMCID: PMC4277605.

32) Bahri BN, Latif Nejad N, Taffazoli M. A Study Of The Effect Of Continuous Professional Support During All Stages Of Maternal Labor On The Level Of Satisfaction Of Primingrevides From The Experience Of Labor. Journal of Shahid Sadoughi University of Medical Sciences and Health. 2003; 11(3): 73-9.

33) Papagni K, Buckner E. Doula Support and Attitudes of Intrapartum Nurses: A Qualitative Study from the Patient's Perspective. J Perin Educ. 2006; 15(1): 11-8. doi: 10.1624/105812406X92949. PMID: 17322940, PMCID: PMC1595283.

34) Larkin P, Begley CM, Devane D. Women's experiences of labour and birth: an evolutionary concept analysis. Midwifery. 2009; 25: e49-e59. doi: 10.1016/j.midw.2007.07.010. PMID: 17996342.

35) Sauls D. Dimensions of Professional Labor Support for Intrapartum Practice. J Nurs Scholarsh. 2006; 38(1): 36-41. doi: 10.1111/j.1547-5069.2006.00075.x. PMID: 16579322. 